\title{
Production of $\alpha$-arbutin from Hydroquinone by Bacillus subtilis TISTR 1248 and Xanthomonas campestris TISTR 2065
}

\author{
Aurasorn Saraphanchotiwitthaya ${ }^{1,2^{*}}$, and Pattana Sripalakit ${ }^{2,3}$ \\ ${ }^{1}$ Department of Pharmaceutical Technology, Faculty of Pharmaceutical \\ Sciences, Naresuan University, Phitsanulok 65000, Thailand \\ ${ }^{2}$ Pharmaceutical Biotechnology Research Unit, Faculty of Pharmaceutical \\ Sciences, Naresuan University, Phitsanulok 65000, Thailand \\ ${ }^{3}$ Department of Pharmaceutical Chemistry and Pharmacognosy, Faculty of \\ Pharmaceutical Sciences, Naresuan University, Phitsanulok 65000, Thailand \\ *Corresponding author. E-mail: aurasorns@nu.ac.th \\ https://doi.org/10.12982/CMUJNS.2020.0060
}

Received: November 13, 2019

Revised: March 3, 2020

Accepted: April 17, 2020

\section{ABSTRACT}

$\alpha$-Arbutin has been used in the cosmetic industry as a skin-whitening agent and in pharmaceutical applications. It can be synthesised by a chemical reaction and a microbial enzyme. This investigation aims to study the production of a-arbutin by microbial fermentation, using whole cells of Bacillus subtilis TISTR 1248 or Xanthomonas campestris TISTR 2065 as a biocatalyst for the transglucosylation reaction between hydroquinone and disaccharide (sucrose and maltose). The amount of a-arbutin in the biotransformation medium under different concentrations of hydroquinone, disaccharides and ascorbic acid was analysed by a high-performance liquid chromatography (HPLC) technique. The results showed that X. campestris TISTR 2065 was more effective than B. subtilis TISTR 1248 on a-arbutin production. The production yield obtained from using sucrose or maltose as a donor was not statistically different. The addition of ascorbic acid resulted in a slight increase in a-arbutin production, spending less time to reach the maximum yield. The maximum production of a-arbutin of approximately $366.33 \pm 21.13 \mathrm{mg} / \mathrm{l}$ (day 12) was achieved from the biotransformation medium containing 5\%w/v hydroquinone and 3\%w/v sucrose together with $0.2 \mathrm{mM}$ ascorbic acid by $X$. campestris TISTR 2065. The elucidation of the $\alpha$-arbutin structure and the biological activity of the biotransformation mixture containing $\alpha$-arbutin needs further investigation. 
Keywords: $\alpha$-Arbutin, Hydroquinone, Bacillus subtilis TISTR 1248, Xanthomonas campestris TISTR 2065

\section{INTRODUCTION}

$\alpha$ - and $\beta$-arbutin are glycosylated hydroquinones that have been used in phytotherapy and the cosmetic industry (Kwiecien et al., 2013). Arbutin has been used for the treatment of urinary tract infection, kidney stones and diuresis (Seo et al., 2012a). The in vivo antitussive activity of arbutin from plants was reported (Strapková et al., 1991). Moreover, the anti-inflammatory effects of arbutin in lipopolysaccharide-stimulated BV2 microglial cells has been shown (Lee et al., 2012). Its antifungal and antioxidant activities have also been proven (Azadbakht et al., 2004). In cosmetics, both the compounds exhibited inhibitory activity on tyrosinase, an enzyme involved in melanin synthesis (Akiu et al., 1988); this enzyme catalyses the hydroxylation of tyrosine to 3-(3, 4-dihydroxyphenyl)alanine (DOPA) and the oxidation of DOPA to dopaquinone (Sugimoto et al., $2005)$. Therefore, $\alpha$ - and $\beta$-arbutin has been wildly used as skin whitening agents (Akiu et al., 1988; Seo et al., 2012b); however, the whitening effect of $\alpha$-arbutin was more than ten times higher than $\beta$-arbutin (Sugimoto et al., 2003). The half maximum inhibitory concentration $\left(\mathrm{IC}_{50}\right)$ of $\alpha$-arbutin on human tyrosinase was $2.0 \mathrm{mM}$ which was more potent than natural arbutin at a concentration higher than $30 \mathrm{mM}$ (Sugimoto et al., 2003; Sugimoto et al., 2007). Recently, the demand for $\alpha$-arbutin has increased significantly in many parts of the world (Liu et al., 2013). $\beta$-arbutin can be extracted from various plant species including bearberry, wheat and pear (Liu et al., 2013), it can be produced via the biotransformation of hydroquinone in in vitro cultures of numerous plant species (Seo et al., 2012a). $\alpha$-Arbutin is mainly produced via microbial biotransformation by using whole cells and carbohydrate-active enzymes (Liu et al., 2013). From previous studies, Bacillus subtilis X-23 was reported as an effective strain among 600 microbial strains screened from soil for $\alpha$-arbutin production by glycosylation of hydroquinone and possessed $\alpha$-amylase activity. Using hydroquinone as a glycosyl acceptor and maltopentaose as the donor reached an $\alpha$-arbutin production yield of $32.4 \%$ (Nishimura et al., 1994). When using Xanthomonas campestris WU-9701 cells as biocatalysts and maltose as a glucosyl donor for $\alpha$-arbutin production, a maximum molar conversion yield of $93 \%$ was obtained (Kurosu et al., 2002). Mutant Xanthomonas maltophilia BT-112 was screened using various mutation techniques. Under optimised conditions, the maximum $\alpha$-arbutin yield of $30.6 \mathrm{~g} / \mathrm{l}(93.6 \%)$ was obtained from $120 \mathrm{mM}$ hydroquinone (Liu et al., 2013).

In this study, we aimed to investigate the efficiency of two culture collections, Bacillus subtilis TISTR 1248 and Xanthomonas campestris TISTR 
2065, from the Thailand Institute of Scientific and Technological Research (TISTR) on the production of $\alpha$-arbutin. Various concentrations of substrates and additives were optimised.

\section{MATERIAL AND METHODS}

\section{Strains and chemicals}

Bacillus subtilis TISTR 1248 and Xanthomonas campestris TISTR 2065 were purchased from the Thailand Institute of Scientific and Technological Research (Pathum Thani, Thailand). $\alpha$-arbutin standards were purchased from the Sigma-Aldrich Chemical Co. (St. Louis, Mo, USA), sucrose and maltose were purchased from Merck (Germany), peptones and yeast extract were purchased from the HiMedia Laboratories (India), and the other reagents were of an analytical grade and purchased from Merck (Germany).

\section{Preparation of medium and stock cell suspensions}

The medium for the cell growth and biotransformation contained $20 \mathrm{~g} / \mathrm{l}$ of sucrose, $10 \mathrm{~g} / \mathrm{l}$ of peptone, $0.5 \mathrm{~g} / \mathrm{l}$ of $\mathrm{MgSO}_{4}, 1 \mathrm{~g} / \mathrm{l}$ of $\mathrm{K}_{2} \mathrm{HPO}_{4}, 1 \mathrm{~g} / \mathrm{l}$ of $\mathrm{KH}_{2} \mathrm{PO}_{4}$, $2 \mathrm{~g} / \mathrm{l}$ of $\mathrm{NaCl}$ and $1 \mathrm{~g} / \mathrm{l}$ of $\mathrm{NaHCO}_{3}$ in distilled water; the $\mathrm{pH}$ was adjusted to 7.0 prior to sterilisation at $121{ }^{\circ} \mathrm{C}$ for $20 \mathrm{~min}$. Stock cell suspensions were prepared by adding a loopful of inoculum from the slant (nutrient agar: $5 \mathrm{~g} / \mathrm{l}$ of peptic digest of animal tissue, $5 \mathrm{~g} / \mathrm{l}$ of $\mathrm{NaCl}, 1.5 \mathrm{~g} / \mathrm{l}$ of beef extract, $1.5 \mathrm{~g} / \mathrm{l}$ of yeast extract and $15 \mathrm{~g} / \mathrm{l}$ of agar) into the medium and cultured under shaking conditions at $25 \pm 2{ }^{\circ} \mathrm{C}, 220 \mathrm{rpm}$ (revolutions per minute) for $48 \mathrm{~h}$ on an orbital shaker (SK3PO, CT laboratory, USA).

\section{Effect of hydroquinone (HQ) concentrations}

A sterile biotransformation medium with an initial volume of $45 \mathrm{ml}$ in 250 $\mathrm{ml}$ Erlenmeyer flasks was inoculated with $5 \mathrm{ml}$ stock cell suspensions of B. subtilis TISTR 1248 or $X$. campestris TISTR 2065. Hydroquinone concentrations at 1,3 and $5 \% \mathrm{w} / \mathrm{v}$ were added into the biotransformation medium and cultured under conditions at $25 \pm 2{ }^{\circ} \mathrm{C}, 220 \mathrm{rpm}$ for 18 days on an orbital shaker. During the biotransformation process, $1.5 \mathrm{ml}$ of the culture medium was collected at $0,7,9,12,15$ and 18 days for the $\alpha$-arbutin analysis. The microbial strain with an appropriate HQ concentration which gave the maximum yield of $\alpha$-arbutin was selected for further experiment.

\section{Effect of carbon sources}

Five $\mathrm{ml}$ of $X$. campestris TISTR 2065 cell suspension was added into a 45 $\mathrm{ml}$ sterile medium contained 5\% w/v HQ and 1, 2 and 3\%w/v sucrose or maltose in $250 \mathrm{ml}$ Erlenmeyer flasks. The biotransformation medium was cultured at 25 
$\pm 2{ }^{\circ} \mathrm{C}, 220 \mathrm{rpm}$ for 18 days. The culture medium was collected at $0,7,9,12,15$ and 18 days to analyse $\alpha$-arbutin. All the experiments were conducted in triplicate.

\section{Effect of ascorbic acid}

Five $\mathrm{ml}$ of $X$. campestris TISTR 2065 cell suspension was added into $45 \mathrm{ml}$ of sterile medium containing 5\%w/v HQ and 3\%w/v sucrose or maltose in 250 $\mathrm{ml}$ Erlenmeyer flasks. Various concentrations of ascorbic acid at 0.1, 0.2 and 0.3 $\mathrm{mM}$ were added into each flask and cultured under the same conditions. The culture medium was collected at $0,7,9,12,15$ and 18 days to analyse $\alpha$-arbutin. All the experiments were conducted in triplicate.

\section{HPLC analysis}

$\alpha$-Arbutin was detected using high-performance liquid chromatography (HPLC) (Shimadzu Corporation, Japan) under the following conditions: column, C18 LunarTM column $(250 \mathrm{~mm} \times 4.60 \mathrm{~mm}, 5 \mu \mathrm{m}$; Phenomenex, CA, USA); temperature, $25 \pm 2{ }^{\circ} \mathrm{C}$; mobile phase, methanol-water $(5: 95$, v/v) with $0.1 \%$ acetic acid v/v; flow rate, $1.0 \mathrm{ml} / \mathrm{min}$; ultraviolet detector, wavelength $280 \mathrm{~nm}$. The calibration curve of $\alpha$-arbutin standard solution at concentrations between $0.5-10.0 \mathrm{~g} / \mathrm{l}$ showed good linearity. The regression line for $\alpha$-arbutin was $\mathrm{y}=18.128 \mathrm{x}-0.2697\left(\mathrm{R}^{2}=0.9997\right)$, where $\mathrm{y}$ is the peak area $\left(\times 10^{-6}\right)$ and $\mathrm{x}$ is concentration of $\alpha$-arbutin $(\mathrm{g} / \mathrm{l})$. Microbial biotransformation reaction to produce $\alpha$-arbutin from hydroquinone, and their chemical structures are shown in Figure 1.
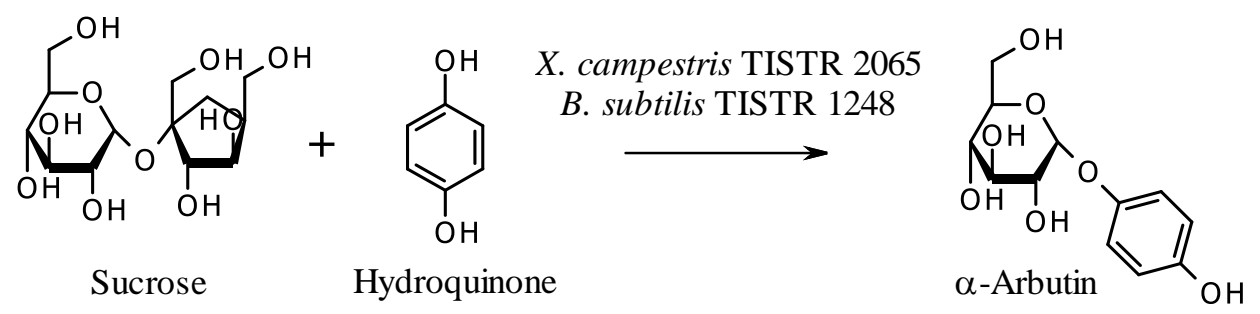

Figure 1. Microbial biotransformation of hydroquinone to $\alpha$-arbutin

\section{RESULTS}

\section{Effects of hydroquinone concentrations}

B. subtilis TISTR 1248 and X. campestris TISTR 2065 were used to produce $\alpha$-arbutin in this study. The effect of different concentrations of hydroquinone in the culture medium was investigated, and the results are shown 
in Figure 2. The results showed that both $B$. subtilis TISTR 1248 and $X$. campestris TISTR 2065 can produce $\alpha$-arbutin. For both strains, using hydroquinone at a high concentration in the culture medium resulted in a higher yield of $\alpha$-arbutin. In the presence of 5\%w/v hydroquinone, $X$. campestris TISTR 2065 produced the maximum yield of $\alpha$-arbutin of approximately $277.66 \pm 12.72$ $\mathrm{mg} / \mathrm{l}$ at day 15 which was more than that produced by B. subtilis TISTR 1248 $(140.52 \pm 10.06 \mathrm{mg} / \mathrm{l}$ at day 15). Therefore, X. campestris TISTR 2065 was selected for further study.

\section{Effects of carbon sources}

The effect of two carbon sources, sucrose and maltose, at different concentrations for $\alpha$-arbutin production by X. campestris TISTR 2065 were studied, the results are shown in Figure 3 . X. campestris TISTR 2065 can produce $\alpha$-arbutin from both sucrose and maltose at all the tested concentrations. A higher yield of $\alpha$-arbutin was produced from using a high concentration of sucrose and maltose. The maximum yield of $\alpha$-arbutin was obtained from using $5 \% \mathrm{w} / \mathrm{v}$ hydroquinone and $3 \% \mathrm{w} / \mathrm{v}$ maltose. However, the production yield of $\alpha$-arbutin obtained using 2 and 3\%w/v sucrose or maltose as a donor was not statistically different.
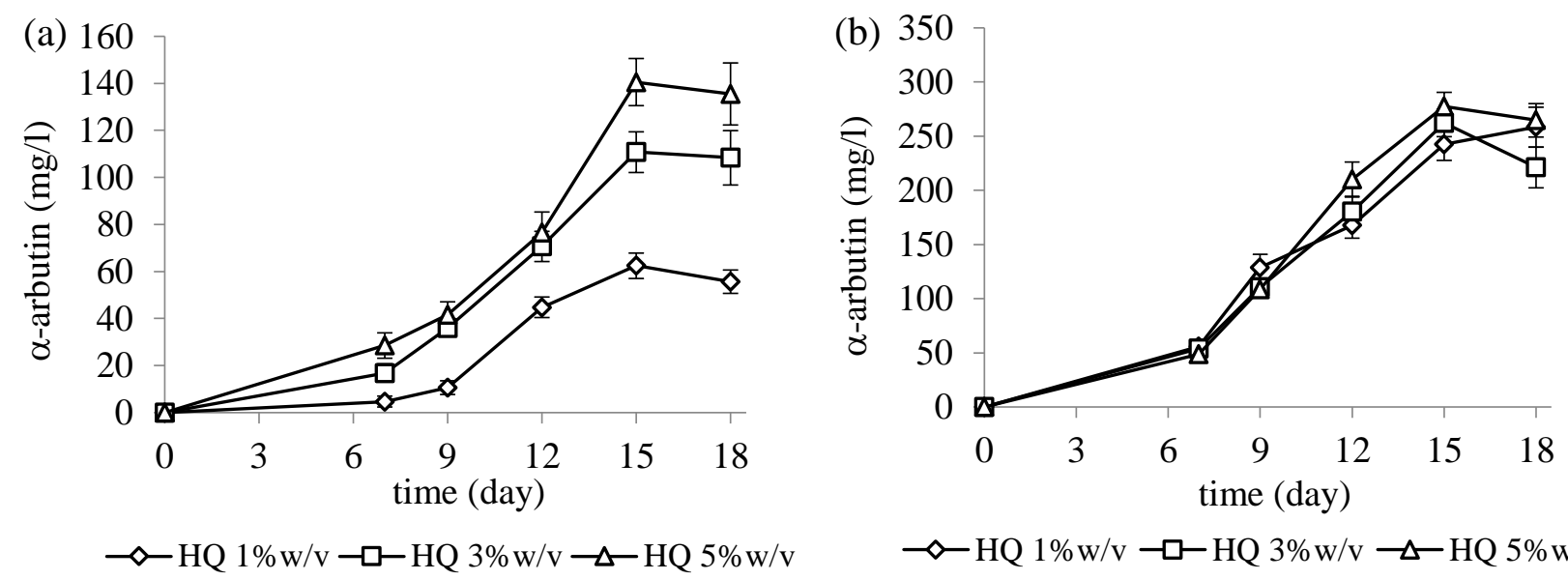

$\neg$ HQ 1\%w/v $\rightarrow-$ HQ 3\%w/v $\rightarrow-$ HQ 5\%w/v

Figure 2. The effect of hydroquinone (HQ) at different concentrations on $\alpha$-arbutin production by (a) Bacillus subtilis TISTR 1248 and (b) Xanthomonas campestris TISTR 2065. The results are represented as the mean $\pm \operatorname{SE}(n=3)$. 

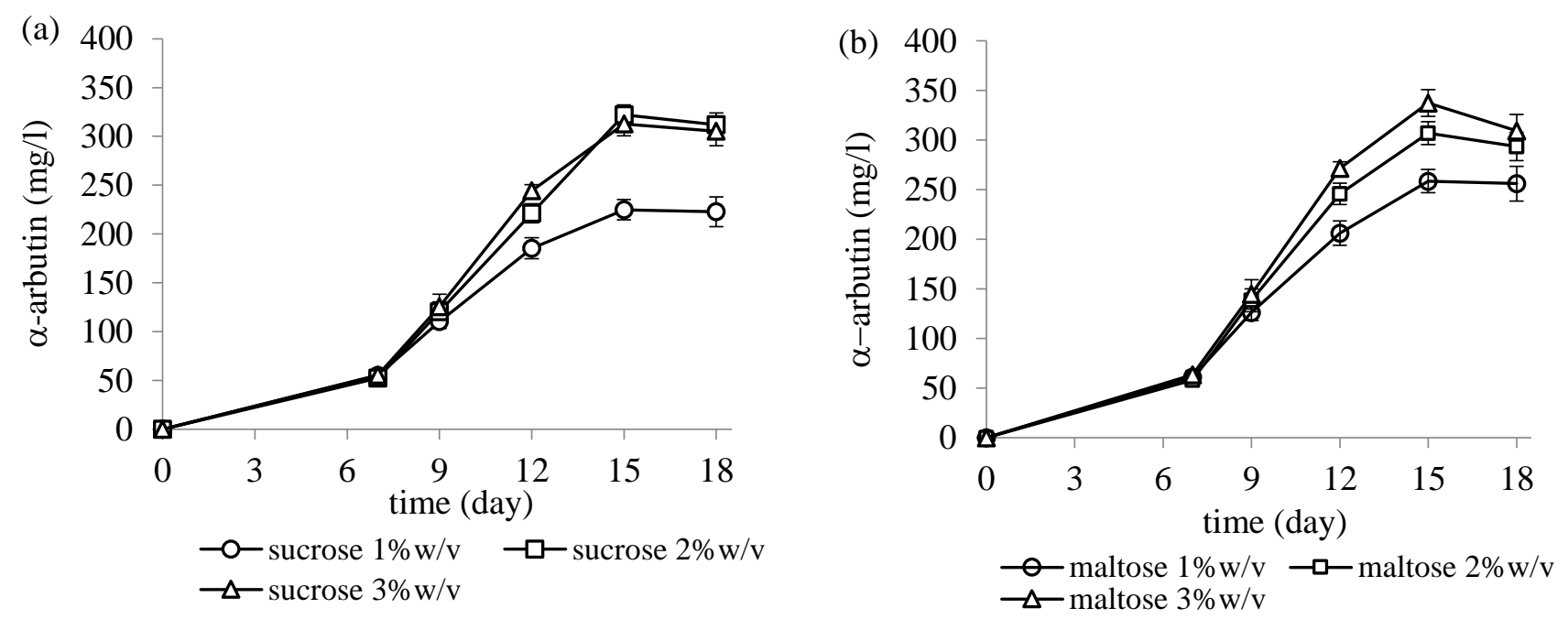

Figure 3. The effect of carbon sources; (a) sucrose and (b) maltose at different concentrations on $\alpha$-arbutin production by Xanthomonas campestris TISTR 2065. The results are represented as the mean \pm SE $(n=3)$.

\section{Effects of ascorbic acid}

The influence of ascorbic acid at different concentrations in the culture medium of $X$. campestris TISTR 2065 for $\alpha$-arbutin production from 5\%w/v hydroquinone and 3\%w/v sucrose or maltose was studied. As shown in Figure 4, the addition of ascorbic acid resulted in a slight increment of $\alpha$-arbutin production reaching a maximum yield in less production time. The maximum yield of $\alpha$ arbutin of approximately $366.33 \pm 21.13 \mathrm{mg} / \mathrm{l}$ (day 12) was obtained from using $5 \% \mathrm{w} / \mathrm{v}$ HQ and $3 \% \mathrm{w} / \mathrm{v}$ sucrose with the addition of $0.2 \mathrm{mM}$ ascorbic acid; however, there was no statistical difference in the $\alpha$-arbutin yield among the different doses of ascorbic acid in both in sucrose and maltose.

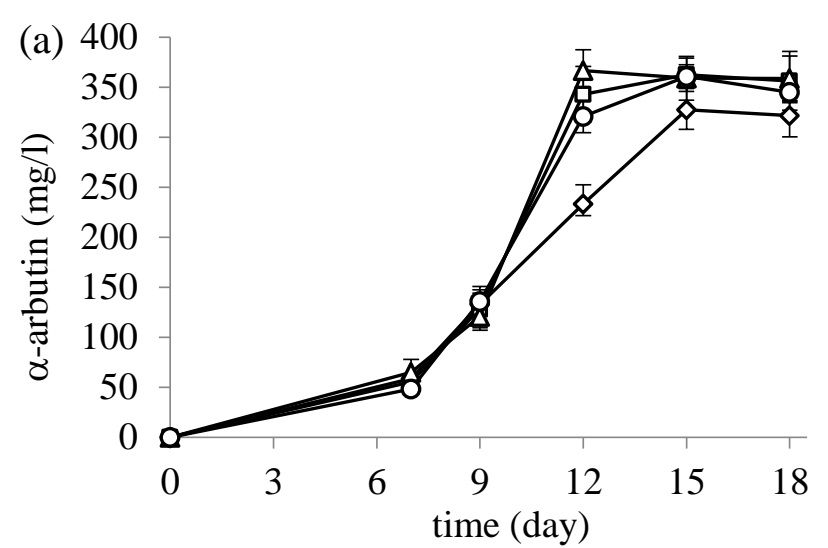

ascorbic acid $\multimap 0-\square-0.1 \multimap-0.2 \multimap 0.3 \mathrm{mM}$

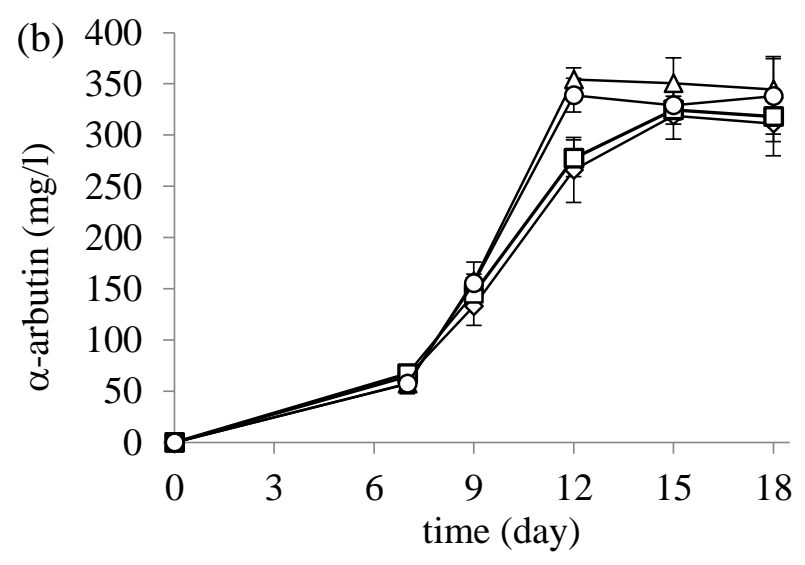

ascorbic acid $\neg 0-\square-0.1 \multimap-0.2 \multimap 00.3 \mathrm{mM}$

Figure 4. The effect of ascorbic acid at different concentrations on $\alpha$-arbutin production from $5 \%$ w/v hydroquinone and (a) $3 \% \mathrm{w} / \mathrm{v}$ sucrose and (b) $3 \% \mathrm{w} / \mathrm{v}$ maltose by Xanthomonas campestris TISTR 2065. The results are represented as the mean \pm SE $(n=3)$. 


\section{DISCUSSION}

$\alpha$-Arbutin is a glycosylated hydroquinone that is mainly used as a powerful skin whitening agent in the cosmetic industry. These compounds can be produced using chemical synthesis, but microbial bioconversion is preferred due to its specificity and eco-friendly environments (Zhu et al., 2018). There have been many previous investigations on the production of $\alpha$-arbutin using whole cells and microbial enzymes. Whole cells such as B. subtilis X-23 (Nishimura et al., 1994), X. campestris Wu-9701 (Kurosu et al., 2002), X. maltophilia BT-112 (Liu et al., 2014) and microbial enzymes such as $\alpha$-glucosidase from $X$. campestris WU-9701 (Sato et al., 2012), dextransucrase from Leuconostoc mesenteroides (Seo et al., 2009) and amylosucrase from Deinococcus geothermalis (Park et al., 2011; Seo et al., 2012b) were used in the studies.

The percentage yield of $\alpha$-arbutin production obtained from the enzymatic and microbial bioconversion of hydroquinone varied from 0.4 to $93.6 \%$. The highest yield of $\alpha$-arbutin (30.6 g/l) was obtained using mutant $X$. maltophilia BT-112 cell with $120 \mathrm{mM}$ HQ and $20 \mathrm{~g} / \mathrm{l}$ of sucrose (Liu et al., 2013). Moreover, $\alpha$-arbutin was effectively produced using lyophilised $X$. campestris WU-9701 cells with $45 \mathrm{mM}$ HQ and $1.2 \mathrm{M}$ maltose, reaching the highest $\alpha$-arbutin yield of $11.4 \mathrm{~g} / \mathrm{l}$ (Kurosu et al., 2002).

Based on the previous researches, we investigated the production of $\alpha$-arbutin using two related microbial strains from the TISTR collection including B. subtilis TISTR 1248 and X. campestris TISTR 2065. It has been found that $X$. campestris TISTR 2065 was more effective than B. subtilis TISTR 1248 in $\alpha$-arbutin production. HQ at a high concentration $(5 \% \mathrm{w} / \mathrm{v}$ or $454 \mathrm{mM})$ resulted in a higher production of $\alpha$-arbutin. A previous study reported that cells undergo apoptosis when using a high concentration of HQ in the medium (Liu et al., 2013); however, this study indicated that X. campestris TISTR 2065 tolerated a high concentration of HQ.

For $\alpha$-arbutin production, carbon sources are necessary nutrients for microbial cell growth and act as a glucosyl donor. A previous study performed by Liu et al. (2013) found that among various carbon sources, sucrose and maltose were suitable for cell growth and $\alpha$-arbutin production by mutant Xanthomonas maltophilia BT-112. Based on these findings, sucrose and maltose were applied as carbon sources in our study. We found that X. campestris TISTR 2065 can employ both sucrose and maltose as a substrate to produce $\alpha$-arbutin and different concentrations of these disaccharides affected the production yield of $\alpha$-arbutin. Although, using maltose provided an $\alpha$-arbutin yield higher than sucrose; however, sucrose was cheaper than maltose resulted in a more cost-effective production. 
Liu et al. (2013) suggested an appropriate ratio of sucrose to HQ at 2:1 (mol) for effective $\alpha$-arbutin production. This ratio was different from our study which was 0.20:1 (mol). The different sucrose to HQ ratio might be appropriate for different strains, leading to their efficiency in the biotransformation process.

Seo et al. (2012a) reported that using ascorbic acid can improve the production yield of $\alpha$-arbutin. In the absence of ascorbic acid, the production yield of $\alpha$-arbutin was significantly low (1.3\%), in the presence of $0.2 \mathrm{mM}$ ascorbic acid, a maximum conversion yield of $\alpha$-arbutin of more than $90 \%$ was obtained. In our study, the addition of ascorbic acid in the biotransformation medium containing $5 \% \mathrm{w} / \mathrm{v}$ hydroquinone and sugar $(3 \% \mathrm{w} / \mathrm{v}$ sucrose or maltose) for $\alpha$ arbutin production by $X$. campestris TISTR 2065 was not in accordance with this previous investigation. We observed a slight increase in the $\alpha$-arbutin yield, requiring less time to reach the maximum yield. Ascorbic acid, acting as an antioxidant, may increase the stability of hydroquinone in the mixture, resulting in the effective production of $\alpha$-arbutin. However, the efficiency of the reactive enzymes and the microbial strain itself remained a significant limitation. The optimization of fermentation conditions might be further investigated to increase the glycosylation activity of wild-type strain.

Recently, whole-cell biocatalysis using engineered $E$. coli together with batch-feeding strategy have been used to increase arbutin production. E. coli cells containing overexpressed amylosucrase, a sucrose-utilizing glycosidase enzyme isolated from $X$. campestris pv. campestris 8004, were used to catalyze glycosylation of hydroquinone. The batch-feeding whole-cell biocatalysis by amylosucrase was designed to eliminate hydroquinone inhibition, resulting enhanced yield (306 mM) and molar conversion rate (95\%) (Zhu et al., 2019a). Process scale-up of these system was evaluated in 5000-L reactor, producing the highest yields (398 mM per 4000-1 reaction volume) and productivities (4.5-4.9 $\mathrm{kg} \mathrm{kl}^{-1} \mathrm{~h}^{-1}$ ) which can be successfully applied for industrial production of $\alpha$-arbutin (Zhu et al., 2019b). For our further investigations, a fed-batch strategy and engineered $E$. coli containing enzyme might be determined to enhance arbutin production.

\section{CONCLUSION}

At present, skin-lightening agents are substantially attractive for both pharmaceutical and cosmeceutical approaches, as a response to the rising demand for treatments of the disorders of skin pigmentations. Therefore, natural arbutin which was synthesised by two wild type microbial strains, $X$. campestris TISTR 2065 and $B$. subtilis TISTR 1248, was investigated in this study. We concluded that $X$. campestris TISTR 2065 was more effective than B. subtilis TISTR 1248 for $\alpha$-arbutin production. Both sucrose and maltose can be used as carbon sources 
for cells growth and acted as a glucosyl donor. The maximum yield of $\alpha$-arbutin $(366.33 \pm 21.13 \mathrm{mg} / \mathrm{l})$ was achieved from the biotransformation medium consisting of $5 \% \mathrm{w} / \mathrm{v}$ hydroquinone and $3 \% \mathrm{w} / \mathrm{v}$ sucrose with $0.2 \mathrm{mM}$ ascorbic acid ( $\mathrm{pH} 7.0$ ) by $X$. campestris TISTR 2065 under conditions of $25 \pm 2{ }^{\circ} \mathrm{C}, 220$ $\mathrm{rpm}$. The elucidation of the $\alpha$-arbutin structure and the biological activity of the biotransformation mixture containing $\alpha$-arbutin needs further investigation. In the future, genetic recombination technology needs to be applied to enhance the expression of $\alpha$-arbutin-producing enzymes to increase $\alpha$-arbutin production.

\section{REFERENCES}

Akiu, S., Suzuki, Y., Fujinuma, Y., Asahara, T., and Fukuda, M. 1988. Inhibitory effect of arbutin on melanogenesis: biochemical study in cultured B16 melanoma cells and effect on the UV-induced pigmentation in human skin. Proceeding of the Japanese Society for Investigative Dermatology. 12: 138139.

Azadbakht, M., Marston, A., Hostettmann, K., Ramezani, M., and Jahromi Maghddam, M. 2004. Biological activity of leaf extract and phenol glycoside arbutin of Pyrus boisseriana Buhse. Journal of Medicinal Plants. 3: 9-14.

Kurosu, J., Sato, T., Yoshida, K., Tsugane, T., Shimura, S., Kirimura, K., et al. 2002. Enzymatic synthesis of $\alpha$-arbutin by $\alpha$ anomer-selective glucosylation of hydroquinone using lyophilised cells of Xanthomonas campestris Wu-9701. Journal of Bioscience and Bioengineering. 93: 328330. https://doi.org/10.1016/S1389-1723(02)80037-8

Kwiecień, I., Szopa, A., Madej, K., and Ekiert, H. 2013. Arbutin production via biotransformation of hydroquinone in in vitro cultures of Aronia melanocarpa (Michx.) Elliott. Acta Biochimica Polonica. 60(4): 865-870.

Lee, H.J., and Kim, K.W. 2012. Anti-inflammatory effects of arbutin in lipopolysaccharide-stimulated BV2 microglial cells. Inflammation Research. 61(8): 817-825. https://doi.org/10.1007/s00011-012-0474-2

Liu, C., Zhang, P., Zhang, S., Xu, T., Wang, F., and Deng, L. 2014. Feeding strategies for the enhanced production of $\alpha$-arbutin in the fed-batch fermentation of Xanthomanas maltophilia BT-112. Bioprocess and Biosystems Engineering. 37(2): 325-329. https://doi.org/10.1007/s00449013-0980-9

Liu, C.Q., Deng, L., Zhang, P., Zhang, S.R., Liu, L., Xu, T., et al. 2013. Screening of high $\alpha$-arbutin producing strains and production of $\alpha$-arbutin by fermentation. World Journal of Microbiology \& Biotechnology. 29(8): 1391-1398. https://doi.org/10.1007/s11274-013-1302-8 
Nishimura, T., Kometani, T., Takii, H., Terada, Y., and Okada, S. 1994. Purification and some properties of $\alpha$-amylase from Bacillus subtilis X-23 that glucosylates phenolic compounds such as hydroquinone. Journal of Fermentation and Bioengineering. 78(1): 31-36. https://doi.org/10.1016/ 0922-338X(94)90174-0

Park, C.S., Seo, D.H., Jung, J.H., and Ha, S.J. 2011. Method for production of $\alpha-$ arbutin using amylosucrase Deinococcus geothermalis. Korea Patent. $1,010,481,480,000$.

Sato, T., Hasegawa, N., Saito, J., Umezawa, S., Honda, Y., Kino, K., and Kirimura, K. 2012. Purification, characterization, and gene identification of an $\alpha$-glucosyl transfer enzyme, a novel type $\alpha$-glucosidase from Xanthomonas campestris WU-9701. Journal of Molecular Catalysis B: Enzymatic. 80: 20-27. https://doi.org/10.1016/j.molcatb.2012.04.014

Seo, D.H., Jung, J.H., Ha, S.J., Cho, H.K., Jung, D.H., Kim, T.J., Baek, N.I., Yoo, S.H., and Park, C.S. 2012b. High-yield enzymatic bioconversion of hydroquinone to $\alpha$-arbutin, a powerful skin lightening agent, by amylosucrase. Applied Microbiology and Biotechnology. 94(5): 1189-1197. https://doi.org/10.1007/s00253-012-3905-7

Seo, D.H., Jung, J.H., Lee, J.E., Jeon, E.J., Kim, W., and Park, C.S. 2012a. Biotechnological production of arbutin ( $\alpha$ - and $\beta$-arbutin), skin-lightening agents and their derivatives. Applied Microbiology and Biotechnology. 95(6): 1417-1425. https://doi.org/10.1007/s00253-012-4297-4

Seo, E.S., Kang, J., Lee, J.H., Kin, G.E., Kim, G.J., and Kim, D. 2009. Synthesis and characterization of hydroquinone glucoside using Leuconostoc mesenteroides dextransucrase. Enzyme and Microbial Technology. 45(5): 355-360. https://doi.org/10.1016/j.enzmictec.2009.07.011

Strapková, A., Jahodář, L., and Nosalová, G. 1991. Antitussive effect of arbutin. Pharmazie. 46: 611-612.

Sugimoto, K., Nishimura, T., and Kurini, T. 2007. Development of $\alpha$-arbutin: production at industrial scale and application for a skin-lightening cosmetic ingredient. Trends in Glycoscience and Glycotechnology. 19(110): 235246. https://doi.org/10.4052/tigg.19.235

Sugimoto, K., Nishimura, T., Nomura, K., Sugimoto, K., and Kuriki, T. 2003. Syntheses of arbutin-alpha-glycosides and a comparison of their inhibitory effects with those of alpha-arbutin and arbutin on human tyrosinase. Chemical and Pharmaceutical Bulletin (Tokyo). 51(7): 798-801. https:// doi.org/10.1248/cpb.51.798

Sugimoto, K., Nomura, K., Nishimura, T., Kiso, T., Sugimoto, K., and Kuriki, T. 2005. Syntheses of $\alpha$-arbutin- $\alpha$-glycosidase and their inhibitory effects on human tyrosinase. Journal of Bioscience and Bioengineering. 99(3): 272-276. https://doi.org/10.1263/jbb.99.272 
Zhu, L., Jiang, D., Zhou, Y., Lu, Y., Fan, Y., and Chen, X. 2019a. Batch-feeding whole-cell catalytic synthesis of $\alpha$-arbutin by amylosucrase from Xanthomonas campestris. Journal of Industrial Microbiology and Biotechnology. 46(6): 759-767. https://doi.org/10.1007/s10295-019-02143$\mathrm{Z}$

Zhu, L., Xu, M., Lu, C., Chen, L., Xu, A., Fang, J., Chen, H., Lu, Y., Fan, Y., and Chen, X. 2019b. Optimization of whole-cell biotransformation for scale-up production of $\alpha$-arbutin from hydroquinone by the use of recombinant Escherichia coli. AMB Express. 9:1-9. https://doi.org/10.1186/s13568-0190820-7

Zhu, X., Tian, Y., Zhang, W., Zhang, T., Guang, C., and Mu, W. 2018. Recent progress on biological production of $\alpha$-arbutin. Applied Microbiology and Biotechnology. 102(19): 8145-8152. https://doi.org/10.1007/s00253-0189241-9 